\title{
High-Efficiency Sub-1 GHz Flexible Compact Rectenna based on Parametric Antenna-Rectifier Co-Design
}

\author{
Mahmoud Wagih ${ }^{1}$, Alex S. Weddell ${ }^{2}$, Steve Beeby ${ }^{3}$ \\ School of Electronics and Computer Science, University of Southampton, UK \\ ${ }^{1}$ mahm1g15@ecs.soton.ac.uk, ${ }^{2}$ asw@ecs.soton.ac.uk, ${ }^{3}$ spb@ecs.soton.ac.uk
}

\begin{abstract}
This work presents a high-efficiency, highsensitivity, compact flexible rectenna based on a high-impedance folded dipole antenna, for sub-1 GHz license-free applications. A voltage-doubler rectifier is studied parametrically, using Electromagnetic-Harmonic Balance co-simulation, to extract the optimal load and source impedances for maximum power conversion efficiency (PCE), using an iterative source and load tuning approach. The proposed antenna is then studied parametrically and designed to directly conjugate the rectifier's impedance eliminating the matching network and its associated losses. The integrated rectenna is ultra-compact $\left(\operatorname{area}=0.0122 \lambda_{0}^{2}\right)$ and is fabricated on a $25 \mu \mathrm{m}$-thick flexible low-cost polyimide substrate. The proposed rectenna achieves a remarkable PCE of $43 \%$ and $83 \%$ at -20 and $-4 \mathrm{dBm}$, respectively. Furthermore, a 1-V DC output is achieved across a $20 \mathrm{k} \Omega$ load (optimal impedance) from $-9 \mathrm{dBm}$ input. The rectenna demonstrates $a-3$ dB $(50 \%$ relative PCE) fractional-bandwidth of $\mathbf{7 . 9 \%}(\mathbf{8 1 3 - 8 8 0}$ $\mathrm{MHz}$ ), covering the $868 \mathrm{MHz}$ license-free band.
\end{abstract}

Keywords - Antenna, Energy Harvesting, Rectenna, Rectifiers, RFID, Wireless Power Transfer.

\section{INTRODUCTION}

Radio Frequency Energy Harvesting (RFEH) and radiative Wireless Power Transfer (WPT), using rectifying antennas (rectennas), present a potential power supply for future autonomous Internet of Things (IoT) devices [1]. In theory, rectennas can achieve a $100 \%$ RF to DC Power Conversion Efficiency (PCE) using ideal diodes and matching components [2]. However, demonstrating high-efficiency rectennas using commercial lumped diodes has always been limited by the rectifier design and the impedance matching approach, where lumped components exhibit internal losses [3], and high-efficiency distributed matching networks need to be implemented on low-loss RF substrates [4].

In order to overcome the additional losses incurred in a matching network, high impedance antennas were proposed to directly match the rectifier [5], [6], [7]. While in [5] a generic high-impedance cross-dipole was able to achieve broadband power conversion for multiple diodes, the high-impedance antenna in [7] achieves a high PCE at low power levels, as it has been optimized for a specific rectifier which has been simulated using its distributed layout model. On the other hand, simulations in [4] demonstrated that the achieved PCE using a lossless matching network, based on numerical optimization of the load and source impedances, cannot be improved using a high-impedance antenna.

With the increase in the popularity of flexible electronic systems as unobtrusive IoT nodes, the demand for flexible and sustainable battery-alternatives has motivated rectenna

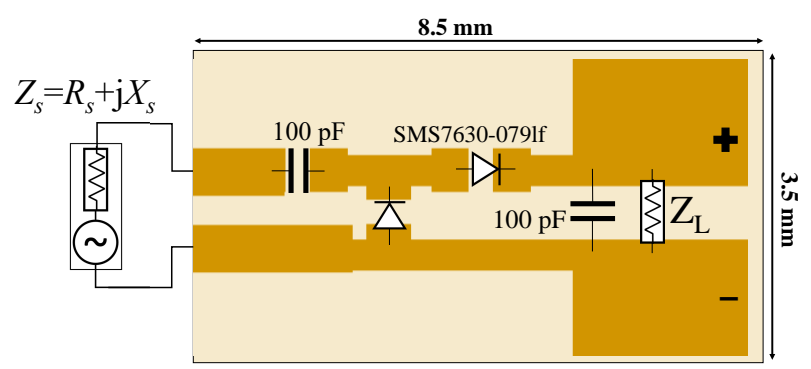

Fig. 1. Layout of the differential voltage doubler rectifier. Parameters $R_{S}$, $X_{S}$ and $Z_{L}$ were optimized using harmonic balance simulation.

development on flexible materials [3], [4], [8]. Although flexible rectennas on paper [3] and textile [9] were reported, their efficiency has been lower than their rigid counterparts implemented on low-loss substrates at the same power levels. Furthermore, the reliance on lumped matching [3] increases the packaging parasitics at higher frequencies, insertion losses, and the overall system complexity. Therefore, designing a high-impedance optimized rectenna without a matching network is essential for compact and efficient RFEH on flexible substrates.

This work presents a flexible high-efficiency rectenna for sub-1 GHz Industrial Scientific Medical (ISM)-band applications. The rectenna achieves the highest reported PCE, of $43 \%$ and $83 \%$ at -20 and $-4 \mathrm{dBm}$, respectively, based on an antenna-rectifier optimization process. The rectenna does not include a matching network and occupies an area of less than $0.0122 \lambda_{0}^{2}$, enabling its seamless integration in wearable applications and battery-free active RF-ID tags. Despite antenna-rectifier co-design being previously presented in [5], [6], [7], the proposed rectenna achieves the highest reported PCE based on an optimized rectifier design through iterative large-signal input s-parameters simulation and parametric high-impedance antenna design.

\section{Rectifier Design AND Simulation}

When designing a high-efficiency rectenna, based on highimpedance antenna-rectifier co-design, the first step is to design and optimize the rectifier separately, extracting its optimal source and load impedances. These values can then be used to guide the antenna design process. Additionally, to improve the accuracy of the simulation models, the feed-lines of the rectifier need to be considered when extracting its input impedance, to account for their impedance transformer 

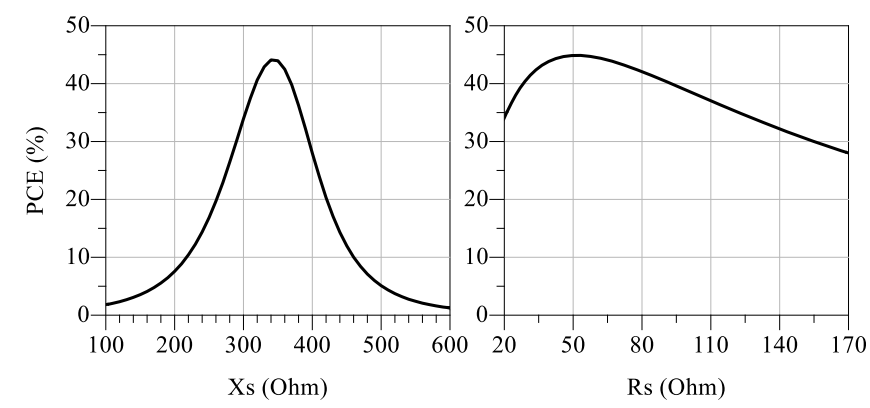

Fig. 2. Simulated PCE of the voltage doubler with variable source input resistance and reactance, at $-20 \mathrm{dBm}$ with a $20 \mathrm{k} \Omega$ load.

effect. To improve the voltage sensitivity, avoiding the need for additional boost converter circuitry and to simplify the system, a voltage-doubler rectifier topology is adopted. The layout of the proposed rectifier, based on the SMS7630-079LF low-barrier Schottky diode, is shown in Fig. 1.

The rectifier has been simulated in Keysight ADS using Harmonic Balance simulation along with Electro-Magnetic (EM) Momentum co-simulation, to factor in the effect of the rectifier's layout impact on the impedance. The Large-Signal S-Parameters (LSSP) of the rectifier were simulated using a source of $Z_{S}=50 \Omega, Z_{S}$ is then varied to approach the simulated values. As the initially extracted $R_{S}$ and $X_{S}$ were obtained using a mismatched source, they do not reflect the LSSP behaviour of the rectifier, nor the small-signal behaviour at the source power level. Therefore, an iterative optimization process has been adopted where the source impedance is varied continuously to achieve the maximum PCE at $-20 \mathrm{dBm}$, ensuring the extracted impedances arise from a rectifier which absorbs most $\left(\Gamma_{\text {Rectifier }}<-10 \mathrm{~dB}\right)$ of the incident power. At higher power levels the rectifier's sensitivity is expected to reduce due to the higher reflection coefficient, where the rectifier's impedance increases with frequency [10].

Fig. 2 shows the variation of the rectifier's PCE for variable $R_{S}$ and $X_{S}$. As the voltage doubler's input impedance is predominantly reactive (capacitive), the source's reactance plays the dominant role in the PCE. The rectifier maintains over 50\% (3 dB bandwidth) of its peak PCE with more than $230 \Omega$ and $170 \Omega$ variation in the input reactance and resistance, respectively, allowing for improved tolerances for antenna fabrication imperfections and simulation errors. The optimal values for the source and load impedances extracted are $R_{S}$ $=50 \Omega, X_{S}=340 \Omega$ and $Z_{L}=20 \mathrm{k} \Omega$. While $Z_{L}$ is dependent on the current drawn by the load and can be considered as a design constraint, the identified optimal load impedance can be used to guide the design of a DC-DC converter circuit of similar input impedance to the optimal $Z_{L}$.

\section{High-Impedance FDA Design ANd Simulation}

A Folded-Dipole Antenna (FDA) can be designed to achieve very high input resistances and reactances, where two shorted folds act as impedance transformers allowing impedances above $100 \Omega$. A FDA has been designed to allow parametric tuning of the antenna's geometry to achieve an

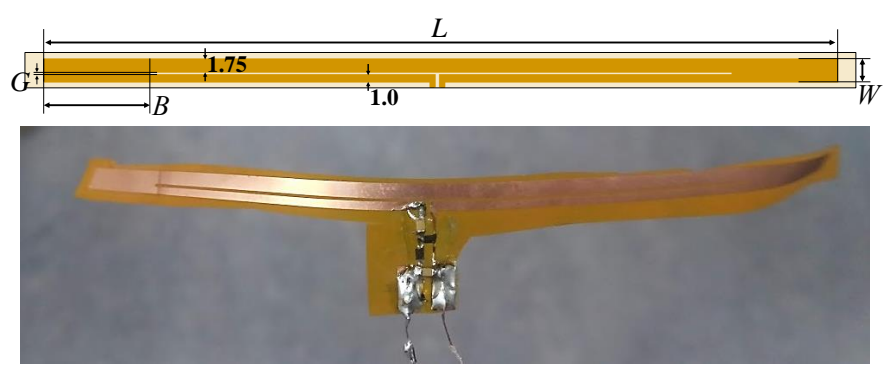

Fig. 3. Layout (top) and photograph (bottom) of the high-impedance FDA, optimized dimensions (in $\mathrm{mm}$ ): $\mathrm{L}=105, \mathrm{G}=0.25, \mathrm{~W}=3, \mathrm{~B}=14.0$.

ideal impedance match to the rectifier's input impedance. Fig. 3 shows the proposed FDA geometry. The parameters $L, G$ and $B$ were varied in the CST model to tune the antenna's input impedance $Z_{\text {ant }}$. While the length $L$ is expected to vary the resonant center frequency, the impedance at resonance is a function of the impedance of the two folded lines. Hence, the gap size can be varied to tune the antenna's impedance both at and near its resonance frequency. The gap, directly influencing the parallel-plate and fringing capacitance of both arms affects mostly $\Im\left\{Z_{a n t}\right\}$. The lines' shorting block width $B$ can be used for finer tuning of $\Re\left\{Z_{\text {ant }}\right\}$ compared to the length $L$.

The three parameter sweeps were performed independently with the starting parameters being $L=100, B=14, G=$ 0.3. Fig. 4 shows the variation in the input impedance across the swept parameters. The optimized FDA selected for this rectenna, whose dimensions are $L=105, B=14$ and $G=$ 0.25 , has an impedance $Z_{\text {ant }}=25+j 340 \Omega$. The antenna was designed to match the rectifier at a lower frequency than its resonance, so that the lower variation in the input impedance does not affect the impedance match, improving the broadband rectenna's response. As seen in Fig. 4-c, a FDA of $L<106.25$ maintains a more stable input impedance around $860 \mathrm{MHz}$, within the rectifier's $Z_{S}$ tolerance for maintaining high PCE (Fig. 2).

The antenna has been fabricated using standard photolithography on a commercial low-cost polyimide copper-laminate, of $25 \mu \mathrm{m}$ thickness [11], Doctor-blading of the photo-resistive ink has been utilized instead of spin-coating to reduce the cost of the fabrication process. The mounted components (diodes and surface-mount capacitors) are encapsulated using UV-curable Glob-Top Epoxy, improving their mechanical reliability and resilience to repeated bending.

\section{Rectenna Wireless Testing}

\section{A. Directional Power-Calibrated Transmitter}

To investigate the integrated rectenna's performance, and validate the rectifier's simulation, a directional $11 \mathrm{dBi}$ antenna has been used to power the rectenna at different power levels. The transmitter antenna and the rectenna were placed at 1-m separation. The received power at the rectenna has been estimated using a standard omnidirectional dipole antenna, as well as calculated using a plane-wave input [12]. Fig. 5 

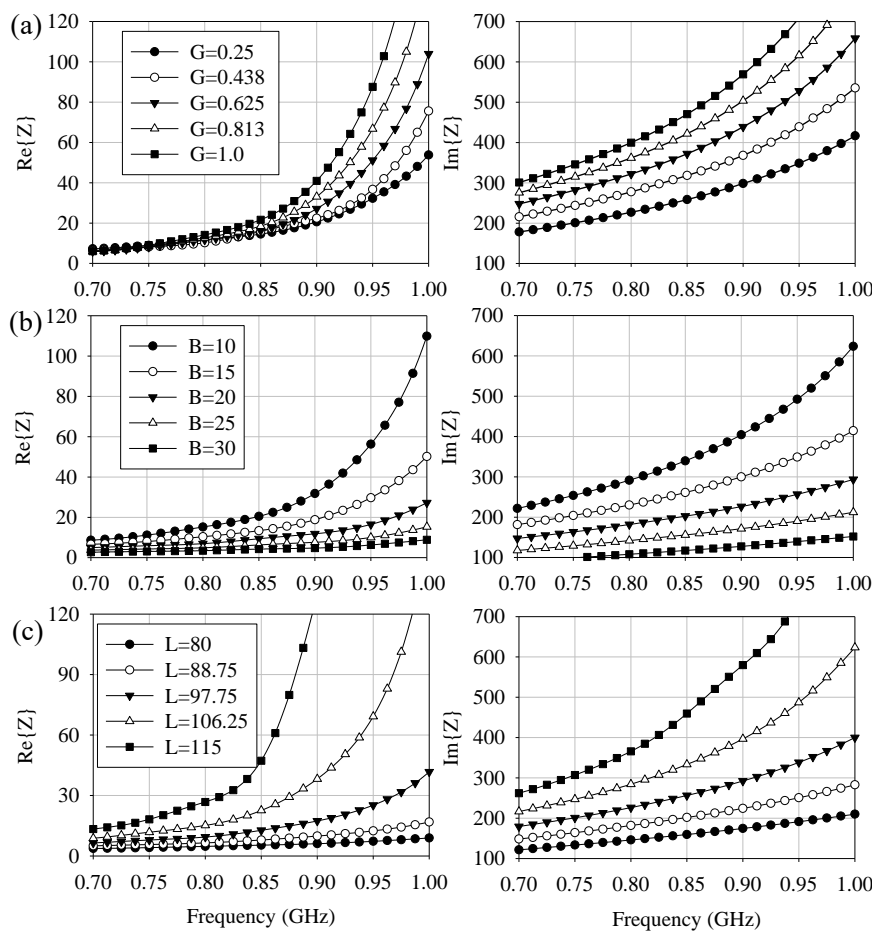

Fig. 4. Simulated real (left) and imaginary (right) input impedance of the FDA for variable $G$ (a), $B$ (b) and $L$ (c).

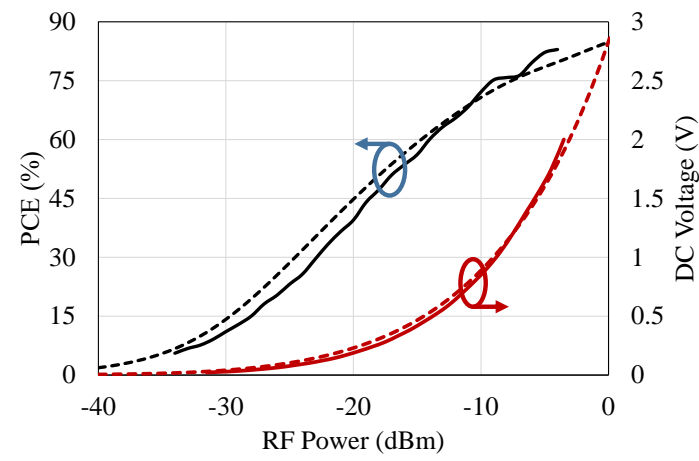

Fig. 5. Measured (solid line) and simulated (dashed line) PCE of the rectenna at $858 \mathrm{MHz}$ with a $20 \mathrm{k} \Omega$ load.

shows the measured PCE and DC voltage across the $20 \mathrm{k} \Omega$ load at variable power levels, from a $860 \mathrm{MHz}$ Continuous Wave (CW) input. The PCE has been calculated using the power estimated RF power using a receiver in presence and in absence of the antenna $\left(\mathrm{PCE}=\frac{P_{D C}}{P_{R F}}\right)$. The simulated values were obtained using an ideal source of $Z=25+j 340 \Omega$.

The frequency of the source has been varied to measure the rectenna's bandwidth. Fig. 6 shows the measured PCE and voltage output of the rectenna at $-18.5 \mathrm{dBm}$ and a $20 \mathrm{k} \Omega$ load. The simulated PCE has been obtained using a broadband source impedance of $Z_{i n}=25+j 340 \Omega$. Therefore, the rectenna's response is more narrow-band due to the increase in the input impedance with frequency (observed in Fig. 4). Nevertheless, the rectenna maintains $50 \%$ of the peak PCE (3 $\mathrm{dB}$ bandwidth) across a 7.9\% fractional bandwidth from (813 to $880 \mathrm{MHz}$ ), which is sufficient for sub-1 $\mathrm{GHz}$ applications

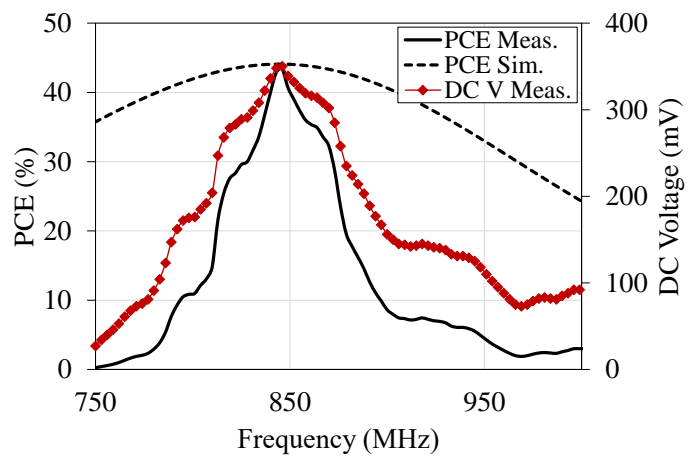

Fig. 6. Simulated and measured PCE of the rectenna at variable frequencies, at $-20 \mathrm{dBm}$ input with a $20 \mathrm{k} \Omega$ load.

Table 1. Comparison of the proposed rectenna with reported rectennas

\begin{tabular}{|c|c|c|c|c|c|}
\hline & $\begin{array}{l}\text { This } \\
\text { work }\end{array}$ & $\begin{array}{l}\text { WPT } \\
2017 \text { [7] }\end{array}$ & $\begin{array}{l}\text { TMTT } \\
2018 \text { [4] }\end{array}$ & $\begin{array}{l}\text { IMS } \\
2019 \text { [9] }\end{array}$ & $\begin{array}{l}\text { TAP } \\
2016 \\
{[10]}\end{array}$ \\
\hline $\begin{array}{l}\text { Freq } \\
(\mathrm{GHz})\end{array}$ & 0.868 & 0.868 & 2.4 & 2.4 & $0.9^{*}$ \\
\hline Matching & $\begin{array}{l}\text { High-Z } \\
\text { antenna }\end{array}$ & $\begin{array}{l}\text { High-Z } \\
\text { antenna }\end{array}$ & $\begin{array}{l}\text { Distrib. } \\
\text { elements }\end{array}$ & $\begin{array}{l}\text { Lumped } \\
\text { L-network }\end{array}$ & $\begin{array}{l}\text { Resistance } \\
\text { compress } \\
\text { network }\end{array}$ \\
\hline Diode & SMS7630 & $\begin{array}{l}\text { HSMS- } \\
2852\end{array}$ & SMS7630 & SMS7630 & SMS7630 \\
\hline $\begin{array}{l}\text { PCE: } \\
-20 \\
\mathrm{dBm}\end{array}$ & $43 \%$ & $33 \%$ & $33.6 \%$ & $15 \%$ & $25 \%$ \\
\hline $\begin{array}{l}\text { PCE: } \\
-10 \\
\mathrm{dBm}\end{array}$ & $70 \%$ & $55 \%$ & $56 \%$ & $35 \%$ & $51 \%$ \\
\hline $\begin{array}{l}\text { Maximum } \\
\text { PCE }\end{array}$ & $83 \%$ & NR & $62 \%$ & $70 \%$ & $67 \%$ \\
\hline $\begin{array}{l}\text { 1-V } \\
\text { Sensitivity } \\
\text { at Z-load }\end{array}$ & $\begin{array}{c}-9 \mathrm{dBm} \\
\text { at } 20 \mathrm{k} \Omega\end{array}$ & NR & $\begin{array}{l}-7 \\
\mathrm{dBm} \dagger \text { at } \\
5 \mathrm{k} \Omega\end{array}$ & $\begin{array}{l}-6.5 \\
\text { dBm†, } \\
\text { load NR }\end{array}$ & $\begin{array}{l}-10 \\
\mathrm{dBm} \dagger \text { at } \\
25 \mathrm{k} \Omega\end{array}$ \\
\hline
\end{tabular}

*Multi-band, only $900 \mathrm{MHz}$ considered for this comparison

$\dagger$ Calculated from the PCE curve. NR: Not reported

and allows the utilization of multi-tone inputs.

Table 1 shows a comparison of the proposed rectenna with high-efficiency low-power rectennas with and without matching networks. It is observed that this work achieves the highest PCE at various power levels, notably at ultra-low power levels $(-20 \mathrm{dBm})$. Compared to other rectennas without matching network [5], [6], the proposed rectenna achieves higher PCE due to being designed specifically for a certain rectifier at low power levels, as well as including the load impedance in the initial iterative antenna-rectifier optimization. While [7] reported rectifier EM co-simulation and optimization of the rectifier design, the diode utilized in this work enables achieving higher PCEs due to its lower turn-on threshold. To add, this rectifier has been optimized for maximum PCE at $-20 \mathrm{dBm}$, at which the input impedance was obtained.

While the proposed high-impedance rectenna achieves the highest PCE, $50 \Omega$ antennas with ideal impedance transformers can achieve equally high PCEs [4]. However, the parasitics of lumped components and the insertion losses of distributed elements matching will result in a non-ideal impedance match. Through careful rectifier EM co-simulation of the rectifier 


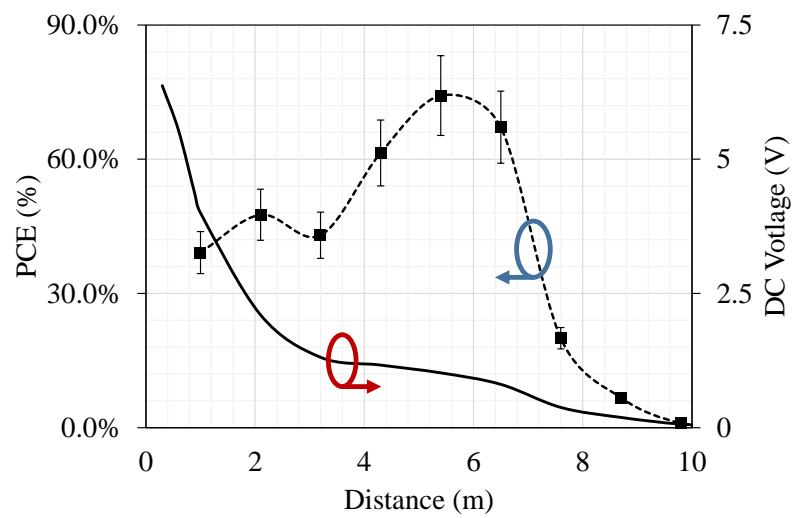

Fig. 7. Measured DC output of the rectenna at varying separation from the 34.77 dBm EIRP Powercast $915 \mathrm{MHz}$ transmitter.

along with antenna-rectifier co-design, the proposed rectenna achieves the highest reported PCE using a commercial diode. The reported rectifiers with higher efficiencies, such as [13], are specifically tuned to sub-100 $\mu \mathrm{W}$ power levels and require many-stage rectifiers with innovative device-biasing techniques, where the PCE still deteriorates significantly at power levels above $-10 \mathrm{dBm}$. Moreover, although [10] achieves higher DC voltage sensitivity, this is due to the resistance compression matching network, allowing higher load impedances without reducing the PCE. However, it cannot be used on a compact flexible system as it significantly increases the system's complexity, as well as reduces the measured PCE due to utilizing non-ideal lumped components [14].

\section{B. Indoor Harvesting from an ISM-Source}

The rectenna is evaluated under real operation conditions using a $3 \mathrm{~W}$ Powercast $915 \mathrm{MHz}$ transmitter in an indoor environment. While the $-20 \mathrm{dBm} 3 \mathrm{~dB}$ power bandwidth does not cover the $915 \mathrm{MHz}$ band (Fig. 6) the rectenna will still operate at $915 \mathrm{MHz}$ with reduced sensitivity due to the impedance mismatch between the antenna and the rectifier at $915 \mathrm{MHz}$. This will result in reduced PCE particularly at lower RF power levels. However, this test is motivated by the availability of an off-the-shelf power transmitter at $915 \mathrm{MHz}$.

The separation between the Powercast transmitter and the rectenna was varied and the DC voltage across the rectenna's $20 \mathrm{k} \Omega$ load has been measured using a multi-meter. Fig. 7 shows the measured DC voltage of the rectenna, the PCE has been calculated using the Friis model with a $34.77 \mathrm{dBm}$ EIRP output. The error bars indicate $\mathrm{a} \pm 1 \mathrm{~dB}$ uncertainty in the incident RF power level. The peak measured PCE is $74 \%$ at a DC voltage of $1-\mathrm{V}$ at $5.4-\mathrm{m}$ separation from the Powercast. Below 2-m separation from the source, the reduced PCE is due to the rectifier operating in the diodes' breakdown region. From 6-m separation and above, the drop in the PCE is due to the propagation losses.

\section{CONCLUSION}

In this paper, a high-efficiency flexible rectenna has been presented. The proposed rectenna achieves a PCE of $43 \%$ and
$83 \%$ at -20 and $-4 \mathrm{dBm}$, respectively, along with $-9 \mathrm{dBm}$ $1-\mathrm{V}$ sensitivity. A voltage doubler rectifier has been simulated and its optimal source and load impedances were extracted, as a result, the proposed rectenna achieves the highest reported PCE based on a commercial Schottky diode.

Through antenna and rectifier parametric co-design, the proposed high impedance FDA eliminates the rectifier matching stage and minimizes the system's complexity while maximizing the efficiency. Through this technique, the potential for high-efficiency wireless-powering of flexible and compact unobtrusive IoT devices is demonstrated.

\section{ACKNOWLEDGEMENT}

This work was supported by the European Commission through EnABLES Project funded under H2020-EU.1.4.1.2.

\section{REFERENCES}

[1] C. R. Valenta and G. D. Durgin, "Harvesting wireless power: Survey of energy-harvester conversion efficiency in far-field, wireless power transfer systems," IEEE Microwave Magazine, vol. 15, 4, pp. 108-120, 2014.

[2] R. Gutmann and J. Borrego, "Power Combining in an Array of Microwave Power Rectifiers," IEEE Trans. Microw. Theory Techn., vol. 27, 12, pp. 958 - 968, 1979.

[3] V. Palazzi, J. Hester, J. Bito, F. Alimenti, C. Kalialakis, A. Collado, P. Mezzanotte, A. Georgiadis, L. Roselli, and M. M. Tentzeris, "A Novel Ultra-Lightweight Multiband Rectenna on Paper for RF Energy Harvesting in the Next Generation LTE Bands," IEEE Trans. Microw. Theory Techn., vol. 66, 1, pp. $366-379,2018$.

[4] S.-E. Adami, P. Proynov, G. S. Hilton, G. Yang, C. Zhang, D. Zhu, Y. Li, S. P. Beeby, I. J. Craddock, and B. H. Stark, "A Flexible 2.45-GHz Power Harvesting Wristband With Net System Output From $-24.3 \mathrm{dBm}$ of RF Power," IEEE Trans. Microw. Theory Techn., 2018.

[5] C. Song, Y. Huang, J. Zhou, P. Carter, S. Yuan, Q. Xu, and Z. Fei, "Matching network elimination in broadband rectennas for high-efficiency wireless power transfer and energy harvesting," IEEE Transactions on Industrial Electronics, vol. 64, 5, pp. 3950 - 3961, 2017.

[6] C. Song, Y. Huang, P. Carter, J. Zhou, S. D. Joseph, and G. Li, "Novel compact and broadband frequency-selectable rectennas for a wide input-power and load impedance range," IEEE Trans. Antennas Propag., vol. 66, 7, pp. 3306 - 3316, 2018.

[7] H. J. Visser, S. Keyrouz, and A. B. Smolders, "Optimized Rectenna Design," Wireless Power Transfer, vol. 2, 1, pp. 44 - 50, 2017.

[8] A. Abdelraheem, M. Sinanis, S. Hameedi, M. Abdelfattah, and D. Peroulis, "A flexible virtual battery: A wearable wireless energy harvester," IEEE Microwave Magazine, vol. 20, 1, pp. 62 - 69, 2019.

[9] D. Vital, S. Bhardwaj, and J. L. Volakis, "A $2.45 \mathrm{GHz}$ RF Power Harvesting System Using Textile-Based Single-Diode Rectennas," in 2019 IEEE MTT-S International Microwave Symposium (IMS), 2019.

[10] C. Song, Y. Huang, P. Carter, J. Zhou, S. Yuan, Q. Xu, and M. Kod, "A novel six-band dual cp rectenna using improved impedance matching technique for ambient rf energy harvesting," IEEE Trans. Antennas Propag., vol. 64, 7, pp. $3160-3171,2016$.

[11] M. Wagih, A. S. Weddell, and S. Beeby, "Millimeter-wave textile antenna for on-body rf energy harvesting in future $5 \mathrm{~g}$ networks," in 2019 IEEE Wireless Power Transfer Conference (WPTC), 2019.

[12] A. Takacs, H. Aubert, S. Fredon, L. Despoisse, and H. Blondeaux, "Microwave Power Harvesting for Satellite Health Monitoring," IEEE Trans. Microw. Theory Techn., vol. 62, 4, pp. 1090-1098, 2014.

[13] A. S. Almansouri, M. H. Ouda, and K. N. Salama, "A cmos rf-to-dc power converter with $86 \%$ efficiency and -19.2-dbm sensitivity," IEEE Trans. Microw. Theory Techn., vol. 66, 5, pp. 2409 - 2415, 2018.

[14] C. Song, Y. Huang, J. Zhou, J. Zhang, S. Yuan, and P. Carter, "A high-efficiency broadband rectenna for ambient wireless energy harvesting," IEEE Trans. Antennas Propag., vol. 63, 8, pp. 3486-3495, 2015. 